Revista Mexicana de Economía y Finanzas Nueva Época

Volumen 14 Número 2, Abril-Junio 2019, pp. 245-259

DOI: https://doi.org/10.21919/remef.v14i2.382

\title{
Negociaciones de máxima probabilidad para juegos cooperativos con fines comerciales
}

\author{
Gerardo Manuell Cid ${ }^{1}$ \\ Instituto Tecnológico Autónomo de México, México \\ Luis V. Montiel \\ Instituto Tecnológico Autónomo de México, México \\ (Recepción: 20/julio/2018, aceptado: 13/diciembre/2018)
}

\section{Resumen}

El objetivo de este trabajo presenta un método para encontrar la estrategia de pagos que maximiza la probabilidad de cerrar una negociación en escenarios cooperativos de utilidad transferible con fines comerciales. Este procedimiento utiliza una herramienta de simulación llamada JDSIM para muestrear el Core del juego y generar funciones empíricas de probabilidad acumuladas de los pagos de cada actor con el fin de establecer la negociación que maximiza el producto de estas funciones. Este método se compara con otros dos ya establecidos en la literatura, llamados Shapley Value (SV) y el centroide del Core, a través de treinta escenarios aleatorios y un caso práctico en los que se observa que este siempre ofrece soluciónes únicas, implementables, y proporciona una justificación racional, a diferencia de los ya mencionados. Hay que considerar que este trabajo únicamente pretende resolver negociaciones en las que la distribución de los pagos permite a todos los actores unirse en una sola coalición, sin embargo, el paradigma presentado se puede adaptar a subcoaliciones de menor tamaño para extender su alcance. Esta solución propone una mejor alternativa a las que se encuentran en la literatura y representa un avance importante en áreas de negociación y teoría de juegos.

Clasificación JEL: C44, Cr71

Palabras clave: Negociación, Shapley Value, Core, JDSIM, Simulación de Juegos

\section{Maximum Probability Negotiations in Cooperative Games with Commercial Purposes}

\section{Abstract}

This work presents a method to find the payment strategy that maximizes the probability of closing a negotiation in cooperative scenarios of transferable utility for commercial purposes. This procedure uses a simulation tool called JDSIM to sample the core of the game and generate cumulative empirical probability functions of the payments of each actor in order to establish the negotiation that maximizes the product of these functions. This method is compared with other two methods already established in the literature, called Shapley Value (SV) and the centroid of the Core, through thirty random scenarios and a practical case in which it is observed that this always offers unique and implementable solutions, and provides a rational justification unlike those already mentioned. It should be borne in mind that this work is only intended to resolve negotiations in which the distribution of payments allows all actors to unite in a single coalition; however, the paradigm presented can be adapted to smaller sub-coalitions to extend its scope. This solution proposes a better alternative to those found in the literature and represents an important advance in areas of negotiation and game theory.

\footnotetext{
${ }^{1}$ Departamento de Ingeniería Industrial y Operaciones - ITAM, Rio Hondo 1, CDMX 01080, México, gmanuellcid@gmail.com,luis.montiel@itam.mx
} 


\section{Abstract}

JEL Classification: $C 44, C 71$

Keywords: Negotiation, Shapley Value, Core, JDSIM, Game simulation

\section{Introducción}

Existe una cantidad reducida de métodos para resolver esquemas de negociación, y casi ninguno de ellos se aplica para solucionar problemas comerciales prácticos debido a la complejidad inherente a este tipo de juegos. Por ello, en este trabajo, se presenta un nuevo mecanismo que utiliza los principios básicos de la Teoría de Juegos para crear una herramienta robusta y de uso práctico, con el fin de resolver negociaciones comerciales (negocios, alianzas, sociedades, etc.) donde $n$ actores requieren ponerse de acuerdo para obtener un beneficio. En particular, se propone una metodología que permita encontrar la estrategia de negociación que maximice los incentivos de los $n$ actores para integrarse en una única coalición en entornos de negociación comercial. Esta metodología provee un esquema de negociación que beneficia a todos los actores o, en su defecto, emite un certificado que determina que no es posible generar una coalición, en la que todos los actores estén dispuestos a participar.

La literatura plantea seis métodos principales para encontrar una distribución de pagos que motiven a los participantes a cerrar una negociación, Von Neumann-Morgenstern solution (von Neumann and Morgenstern, 1944), Shapley Value (Shapley, 1953), el Core (Shapley, 1952), Bargaining Set (Aumann and Maschler, 1961), el Kernel (Davis and Maschler, 1965) y el Nucleolo (Schmeidler, 1969).

De estos métodos, Shapley Value (SV) y el Core destacan por ser los más populares debido a su intuición. En el caso de SV, el método calcula el pago promedio que cada jugador recibe de acuerdo con todos los posibles pagos que podría recibir en cada una de las permutaciones de la coalición en la que participan todos los jugadores (Gran Coalición, denominada como N). Estos pagos se calculan en función de las contribuciones marginales de cada jugador. A diferencia de SV, el Core se puede describir como el set de todas las posibles negociaciones que son aceptables para todos los jugadores, considerando todas las subcoaliciones que se pueden formar entre los $n$ participantes.

Desafortunadamente, ni SV ni el Core ofrecen soluciones confiables. En el caso de SV, la estrategia de negociación no siempre es implementable; es decir, en ciertas ocasiones, SV propone una distribución de pagos en la que al menos un actor recibe menos beneficios que si optara por no participar en la Gran Coalición. En estos casos, la propuesta de SV puede llevar a inferir que la cooperación entre los $n$ jugadores no es posible, ya que no existen incentivos suficientes que aseguren que los actores se mantendrán en la Gran Coalición. En el caso del Core, el problema estriba en que, por lo general, no existe una estrategia única. De modo que, aunque todas las estrategias dentro del Core proveen los incentivos para que todos los actores participen en la Gran Coalición, resulta extremadamente difícil encontrar una distribución de los beneficios que sea justa a los ojos de todos los participantes. Un ejemplo se da con el centroide del Core, que, aunque es una estrategia única e implementable, no existe un argumento claro para justificar que dicha distribución de pagos deba ser aceptada de igual modo por todos los $n$ miembros de la Gran Coalición.

Otros métodos menos populares, como Von Neumann-Morgenstern solution utiliza el concepto de dominación (von Neumann and Morgenstern, 1944) para definir un set de pagos $\mathbf{S}$ en donde una distribución de pagos " $x$ " domina a otra " $y$ " cuando cada uno de los miembros de la coalición obtiene un pago mayor dentro de la distribución de pagos " $x$ " al que obtendría con la distribución " $y$ ". Esto implica que ninguna distribución de pagos 
" $x$ " contenida en $\mathbf{S}$ es dominada por otra distribución " $y$ " contenida en $\mathbf{S}$. Y que toda distribución de pagos " $x$ " no contenida en $\mathbf{S}$ está dominada por alguna distribución " $y$ " contenida en $\mathbf{S}$. Aunque el problema de este concepto radica en que no es una solución fácil de calcular ni tampoco hay certeza de que el set $\mathbf{S}$ de un juego exista (Lucas, 1969) o sea único (Lucas, 1992).

Por otro lado, soluciones como el Kernel y el Nucleolo -además de ser complejas de calcular- pertenecen a la familia del Bargaining Set, que representan soluciones alternativas que surgen a partir del concepto de la solución de Von Neumann-Morgenstern, con la finalidad de definir un conjunto de soluciones estables en el sentido de que ningún jugador tenga un incentivo a salirse de una coalición, y por ende, pueda contrarrestar cualquier objeción de otro jugador que pertenezca a la coalición con una contra-objeción. No obstante, como explican Aumann y Drèze, la familia de soluciones del Bargaining Set se calcula a partir de una subcoalición determinada, por lo que sus soluciones no involucran necesariamente a la Gran Coalición, que es el principal interés de este trabajo. Por estas razones, el enfoque consistirá en comparar este método con las soluciones propuestas por SV y el Core, y se considerarán los otros métodos en un futuro cuando se trabaje en optimizar estrategias de negociación en escenarios en los que la Gran Coalición no es implementable.

\section{Negociaciones comerciales}

En esta sección, se enfatiza el carácter de las negociaciones comerciales para distinguirlas de aquellas con fines sociales. La diferencia entre estas se refiere al punto de interés de cada una dentro del proceso de negociación. Mientras que en negociaciones comerciales, los participantes del juego pueden decidir si actúan de forma cooperativa generando un beneficio grupal o ejecutan el proyecto por su cuenta obteniendo un beneficio particular, en negociaciones sociales, se requiere forzosamente que un cierto número de jugadores formen una coalición con suficientes votos para llevar a cabo el proyecto y distribuirse el beneficio, en tal caso, los jugadores que quedan fuera de la coalición ganadora no reciben ningún beneficio, ni tienen posibilidad de generar algún beneficio de modo particular. Por esta razón, existe una mayor prioridad en la forma en la que cada jugador decide unirse o no a una coalición.

Dentro de las negociaciones comerciales, es natural asumir que la utilidad es transferible, ya que equivale a generar transferencias monetarias entre los distintos actores para obtener el máximo beneficio para todos. De modo similar, se asume información perfecta, ya que la información imperfecta reduce la posibilidad de crear la Gran Coalición, lo que resulta en detrimento de todos los participantes.

Dentro del contexto de las negociaciones comerciales, se define una Negociación Implementable como:

Definición 1 Llámese Negociación Implementable a toda distribución de pagos para $\mathbf{N}$ tal que cada subcoalición obtiene un beneficio mayor o igual al que tendría si actuara por su cuenta.

De modo que el Core de una negociación es el conjunto de todas las negociaciones implementables. Gillies (1959) presenta una definición equivalente descrita como "El espacio $n$-dimensional delimitado por todas las negociaciones que satisfacen las restricciones de la distribución del valor del juego entre los $n$-actores". Para ambas definiciones, el interés de este trabajo consiste en encontrar una distribución de pagos que sea implementable, única, y que maximice la intención de todos los involucrados, en participar, para garantizar el máximo beneficio posible. Dicho lo anterior, se considera que esta metodología es una alternativa más robusta a alternativas anteriores por los siguientes dos puntos:

1. La negociación propuesta para $\mathbf{N}$ siempre está contenida dentro del Core y, por ende, cada jugador siempre recibe un beneficio mayor o igual al que obtendría si 
actuara por su cuenta, de tal manera que la negociación es implementable para negociaciones comerciales.

2. La negociación propuesta maximiza la probabilidad de que los jugadores cooperen entre sí, y siempre es mayor o igual a la probabilidad de las negociaciones que ofrecen SV y el Centroide del Core.

\section{Métodos existentes en la literatura}

\subsection{Shapley Value}

Shapley Value es un número que busca representar el valor de un juego esencial para un jugador, sintetizando todas las distintas alternativas a las que se puede enfrentar en un juego definido en forma de función característica (von Neumann and Morgenstern, 1944). El jugador puede actuar por cuenta propia, cooperar con uno u otro jugador o colaborar con todos los jugadores para formar $\mathbf{N}$. Además, tiene la propiedad de ser el único valor que cumple con los siguientes 3 axiomas Shapley, 1953, p.309:

1. Simetría: El valor de un juego es esencialmente una propiedad del juego abstracto.

2. Eficiencia: El valor calculado del juego representa una distribución del valor total del juego.

3. Ley de Agregación: Para combinar dos juegos independientes, sus valores se deben sumar jugador por jugador.

Al cumplir con estos 3 axiomas, Shapley Value permite evaluar de forma a priori el valor esperado de cada jugador de participar en el juego, considerando que todos los demás jugadores se unen a la coalición en forma completamente aleatoria, pero igualmente probable. De modo que cada jugador demanda y recibe un pago de acuerdo con su contribución marginal promedio dentro del valor de cada coalición (Shapley, 1953).

Cabe destacar que Shapley Value utiliza el concepto de función característica $v: \mathbb{B}^{\mathrm{n}} \rightarrow$ $\mathbb{R}$ desarrollado por Von Neumann y Morgenstern para representar el valor de un juego, con base en 3 propiedades fundamentales Von Neumann y Morgenstern, 1944, p.241:

$$
\begin{gathered}
v(\emptyset)=0, \\
v(-\mathbf{S})=-v(\mathbf{S}) \quad \text { para } \quad \mathbf{S} \subset \mathbf{N}, \\
v(\mathbf{S} \cup \mathbf{T}) \geq v(\mathbf{S})+v(\mathbf{T}) \quad \text { si } \quad \mathbf{S} \cap \mathbf{T}=\emptyset \quad \text { para } \quad \mathbf{S}, \mathbf{T} \subset \mathbf{N} .
\end{gathered}
$$

De estas tres propiedades, la ecuación 1 se refiere a que el valor de un juego con cero jugadores tiene un valor de cero; la ecuación 2 consiste en que el valor de la función característica evaluada en el complemento del set $\mathbf{S}(-\mathbf{S})$ es el mismo que el complemento de la función característica evaluada en $\mathbf{S}\left(-v(\mathbf{S})=\sum_{i \in \mathbf{N}} v(i)-\sum_{i \in \mathbf{S}} v(i)\right)$; finalmente, la ecuación 3 hace referencia a la necesidad de que la función característica sea superaditiva y obliga a que el valor de cualquier coalición genere, por lo menos, el mismo valor que el que obtiene cada jugador si actúa por su cuenta cuando se evalúan dos conjuntos disjuntos $\mathbf{S}$ y $\mathbf{T}$ con $\mathbf{S}, \mathbf{T} \subset \mathbf{N}$. Una ventaja de este nuevo método es que se puede aplicar aun cuando la función característica no es superaditiva, permitiendo encontrar negociaciones en escenarios más generales que los permitidos por SV.

\subsection{El Core}

La idea del Core parte esencialmente de la Curva de Contrato (Edgeworth, 1881), en el sentido de que todos aquellos puntos que componen la curva y proponen una distribución de los bienes entre los actores de la economía son equivalentes a las negociaciones que componen el Core de un juego. Estos conceptos son equivalentes porque se refieren a todas 
aquellas negociaciones en las que ningún jugador puede beneficiarse sin necesariamente perjudicar al otro (son puntos eficientes en el sentido de Pareto), y la negociación final que ocurrirá entre los jugadores dependerá completamente del poder de negociación que tenga cada cada uno.

No obstante, la noción moderna del Core fue desarrollada por Shapley (Shapley, 1952) y Gillies (Gillies, 1959) que definen el Core como "el conjunto de negociaciones que no pueden ser mejoradas por otras negociaciones fuera del set" y cuenta con las siguientes características:

1. Asignaciones Eficientes: La suma de los pagos de cualquier negociación que compone el set siempre es igual al valor total del juego.

2. Set Cerrado y Convexo: El set contiene todos sus puntos límite y es convexo.

Un tema específico de interés para este trabajo es la caracterización o mapeo del Core de un juego. Para ello, se decidió utilizar el método JDSIM (Montiel and Bickel, 2013) por ser el único método que permite mapear adecuadamente y en un tiempo razonable un espacio $n$ dimensional al irse incrementado el número de dimensiones. Otros métodos, como aceptación-rechazo (von Neumann, 1963), mapeo por combinaciones convexas de los vértices del politopo (Devroye, 1986), y mapeo a través de la descomposición del área de interés (Rubin, 1984), mostraron volverse lentos e inestables a medida que se incrementaba $n$.

Esta caracterización es fundamental porque permite conocer y explorar el Core de un juego más allá de las restricciones de la distribución de los pagos para ofrecer una solución de mayor calidad.

\subsection{Fallas y Aciertos de SV y el Core: Ejemplo Ilustrativo}

A continuación, se presenta un ejemplo para ilustrar las soluciones y respectivas fallas que ofrecen SV y el Core, dentro de un problema de distribución de costos.

Supóngase un juego de 3 jugadores (a), (b), (c) que están ubicados en el punto O de la Figura 1. El jugador (a) necesita desplazarse hasta el punto A, el jugador (b) al punto $\mathrm{B}$, el jugador (c) al punto $\mathrm{C}$.

Figura 1. Representación gráfica de un juego entre los jugadores (a), (b), y (c).

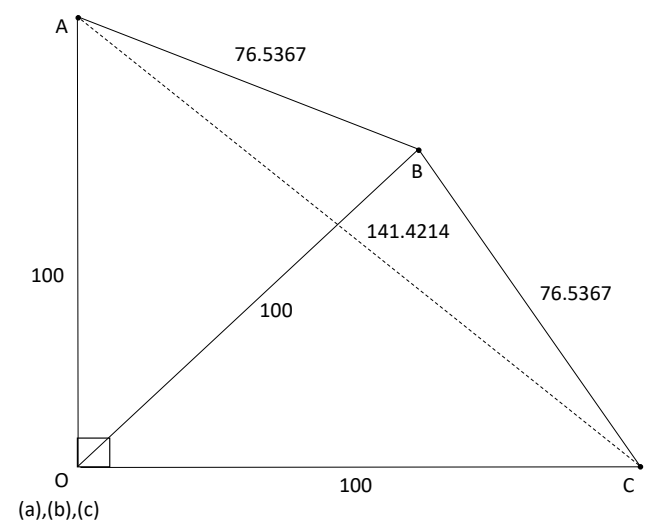

Asúmase un costo asociado de $\$ 1$ por unidad de distancia, de tal manera que si el jugador (a) necesita viajar 100 unidades, entonces, el costo asociado con desplazarse 
esa distancia sería $\$ 100$. Siguiendo esta misma lógica, cada uno de los 3 jugadores está dispuesto a pagar $\$ 100$ para desplazarse a su destino final (el jugador (a) al punto A, el jugador (b) al punto B y el jugador (c) al punto C), y en consecuencia, el costo total del juego será $\$ 300$. Sin embargo, si los 3 jugadores deciden cooperar entre sí, podrían viajar juntos y tomar una ruta distinta, por ejemplo, la ruta $\overrightarrow{O A}(\$ 100)$ más $\overrightarrow{A B}(\$ 76.5)$ más $\overrightarrow{B C}(\$ 76.5)$, que es la ruta que minimiza tanto la distancia como el costo, comparado con el resto de las rutas que pasan por los puntos O, A, B y C. Nótese que para cualquier coalición, el costo total siempre es menor o igual que la suma de los costos cuando todos los jugadores actúan por su cuenta (Tabla 1).

Tabla 1. Costo máximo por esquema de cooperación.

\begin{tabular}{lc}
\hline \hline Coalición & Valor del Juego \\
\hline (a) & $\$ 100$ \\
$(\mathrm{~b})$ & $\$ 100$ \\
$(\mathrm{c})$ & $\$ 100$ \\
$(\mathrm{a}, \mathrm{b})$ & $\$ 176.5$ \\
$(\mathrm{a}, \mathrm{c})$ & $\$ 241.4$ \\
$(\mathrm{~b}, \mathrm{c})$ & $\$ 176.5$ \\
$(\mathrm{a}, \mathrm{b}, \mathrm{c})$ & $\$ 253$ \\
\hline \hline
\end{tabular}

El método SV consiste en calcular la distribución de los pagos en todas las permutaciones posibles de la coalición de 3 jugadores, asumiendo que cada uno se unirá a la coalición en el orden definido en la permutación. Por ejemplo, en la coalición ABC, el jugador A es el primero en unirse, después el jugador B y finalmente el jugador C. Así pues, el primer jugador en unirse a la coalición estará dispuesto a pagar la misma cantidad que habría pagado si hubiera decidido viajar solo. El segundo jugador pagará el remanente del valor de la coalición formada por estos 2 jugadores y el tercer jugador pagará el remanente del valor de la coalición formada por los 3 jugadores, como se muestra en la Tabla ??. Por ejemplo, si (a) se une primero, pagará \$100, que es la misma cantidad que habría pagado si hubiera viajado solo; si (b) se une después, entones (b) pagará únicamente el remanente del costo adicional que implicaría formar la coalición $(a, b)$ que equivale a $\$ 176.5-\$ 100=\$ 76.5$. De tal manera que si (c) decide participar, pagaría el remanente del costo de formar la coalición (a,b,c), es decir, $\$ 253-\$ 176.5=\$ 76.5$.

De esta forma, SV intenta modelar todos los distintos escenarios de negociación que podrían surgir entre los 3 jugadores en función del orden en el que cada jugador se une a la coalición y, con base en ello, calcula el pago promedio esperado de cada jugador. En otras palabras, SV estima el pago esperado de cada jugador considerando todos los escenarios de negociación en los que un jugador se puede ver involucrado, como se muestra en la Tabla 2.

Tabla 2. Pagos para todas las permutaciones para un juego de tres actores.

\begin{tabular}{lccc}
\hline \hline Coalición & $(\mathrm{a})$ & $(\mathrm{b})$ & $(\mathrm{c})$ \\
\hline$(\mathrm{a}, \mathrm{b}, \mathrm{c})$ & $\$ 100$ & $\$ 76.5$ & $\$ 76.5$ \\
$(\mathrm{a}, \mathrm{c}, \mathrm{b})$ & $\$ 100$ & $\$ 11.7$ & $\$ 141.4$ \\
$(\mathrm{~b}, \mathrm{a}, \mathrm{c})$ & $\$ 76.5$ & $\$ 100$ & $\$ 76.5$ \\
$(\mathrm{~b}, \mathrm{c}, \mathrm{a})$ & $\$ 76.5$ & $\$ 100$ & $\$ 76.5$ \\
$(\mathrm{c}, \mathrm{a}, \mathrm{b})$ & $\$ 141.4$ & $\$ 11.7$ & $\$ 100$ \\
$(\mathrm{c}, \mathrm{b}, \mathrm{a})$ & $\$ 76.5$ & $\$ 76.5$ & $\$ 100$ \\
\hline SV & $\$ 95.1$ & $\$ 62.8$ & $\$ 95.1$ \\
\hline \hline
\end{tabular}

Nótese que el costo total de todas las posibles coaliciones entre los 3 jugadores debe ser igual al costo total previamente calculado de $\$ 253$ en la Tabla ??. De acuerdo con los 
valores obtenidos con este método, los jugadores (a) y (c) pagan $\$ 95.1$ cada uno, mientras que el jugador (b) paga $\$ 62.8$.

Otra metodología que permite modelar este problema y buscar una solución al juego es definiendo el Core del juego. El Core consiste en el conjunto de todas las posibles negociaciones que pueden ocurrir entre los jugadores (a),(b) y (c) y está definido por las siguientes restricciones: 2

$$
\begin{gathered}
a \leq 100, \\
b \leq 100, \\
c \leq 100, \\
a+b \leq 176,5, \\
a+c \leq 241,4, \\
b+c \leq 176,5, \\
a+b+c=253 .
\end{gathered}
$$

A partir de estas restricciones, se puede observar que existe un infinito de negociaciones posibles que podrían ocurrir entre los integrantes de $\mathbf{N}$, como por ejemplo: $x=(\$ 100, \$ 70, \$ 83), x=(\$ 80, \$ 90, \$ 83)$ y $x=(\$ 95, \$ 60, \$ 98)$, entre otras. Como el Core del juego no es único, la dificultad consiste en elegir una negociación de este infinito de negociaciones posibles y proponerla como una solución al juego junto con un argumento que la justifique.

Considérese ahora modificar el ejemplo en la Figura 1 cambiando la segunda restricción. Esto implica cambiar la distancia que recorrerá b a $b \leq 50 \sqrt{2}$ obteniendo así el juego mostrado en la Figura 1.

Figura 2. Representación gráfica del juego modificado.

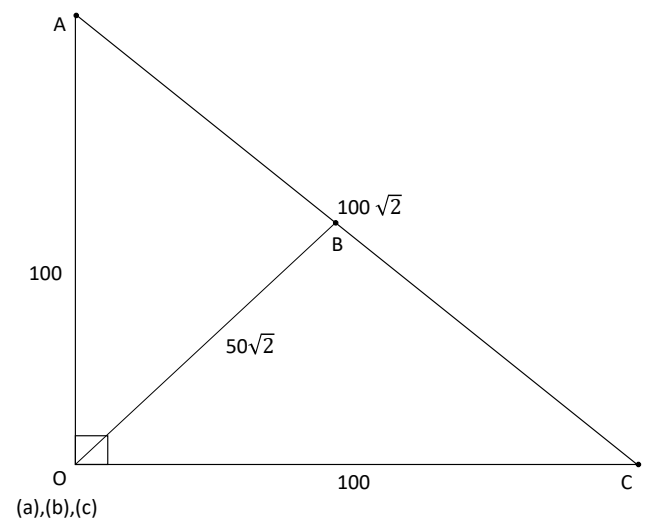

La Tabla 3 muestra los nuevos valores para el cálculo de las permutaciones de SV, y el promedio de estas. 
Tabla 3. Pagos modificados para todas las permutaciones de un juego de tres actores.

\begin{tabular}{lccc}
\hline \hline Coalición & $(\mathrm{a})$ & $(\mathrm{b})$ & $(\mathrm{c})$ \\
\hline$(\mathrm{a}, \mathrm{b}, \mathrm{c})$ & $\$ 100$ & $\$ 41.4$ & $\$ 100$ \\
$(\mathrm{a}, \mathrm{c}, \mathrm{b})$ & $\$ 100$ & $\$ 0$ & $\$ 141.4$ \\
$(\mathrm{~b}, \mathrm{a}, \mathrm{c})$ & $\$ 70.7$ & $\$ 70.7$ & $\$ 100$ \\
$(\mathrm{~b}, \mathrm{c}, \mathrm{a})$ & $\$ 100$ & $\$ 70.7$ & $\$ 70.7$ \\
$(\mathrm{c}, \mathrm{a}, \mathrm{b})$ & $\$ 141.4$ & $\$ 0$ & $\$ 100$ \\
$(\mathrm{c}, \mathrm{b}, \mathrm{a})$ & $\$ 100$ & $\$ 41.4$ & $\$ 100$ \\
\hline SV & $\$ 102$ & $\$ 37.4$ & $\$ 102$ \\
\hline \hline
\end{tabular}

Como se observa en la Tabla 3, la negociación que propone Shapley Value no es implementable porque no se encuentra contenida dentro del Core del juego. Este problema surge a partir de la lógica en la que SV modela los escenarios de negociación que podrían ocurrir entre los miembros de $\mathbf{N}$, puesto que al ir definiendo los pagos de cada jugador dentro del juego, solamente con base en los jugadores que ya forman parte de la coalición, se está perdiendo de vista a los jugadores que todavía no se unen. Esto causa que cuando el último jugador se integra a la coalición, únicamente le corresponde pagar el "remanente" del costo total que aún no ha sido cubierto, ocasionando así que SV ofrezca negociaciones que violan las restricciones del Core. Sin embargo, sí existe una estrategia de negociación implementable $(\$ 100, \$ 41,4, \$ 100)$ que SV no es capaz de encontrar. Por esta razón, es necesario desarrollar mecanismos más confiables que ofrezcan soluciones únicas e implementables. Y que, en caso de no encontrar una solución implementable, genere un certificado que garantice que no existe ninguna estrategia de negociación posible.

\section{Estrategia de Máxima Probabilidad}

Con la finalidad de encontrar la negociación de máxima probabilidad para un juego cooperativo entre $n$-actores, se propone la siguiente metodología:

1. Definir el valor de los pagos que reciben las $2^{n}-1$ coaliciones que se pueden formar entre los $n$ actores.

2. Definir el Core del juego C.

3. Generar una muestra representativa (M) del Core del juego uniformemente distribuida en términos monetarios.

(a) Si el Core está vacío, la metodología indica que no existe negociación alguna que pueda cumplir con las restricciones del Core del juego.

(b) Si el Core es único, la negociación encontrada es la negociación de máxima probabilidad $x^{*}$

(c) Si el Core no está vacío, ir al Paso 4.

4. Obtener las funciones empíricas de probabilidad acumuladas $F_{i}\left(x_{i}\right)$ de cada jugador y definir la Función de Probabilidad de Ocurrencia $(P P)$.

5. Establecer el problema que maximice la función $P P$ y calcular la negociación de máxima probabilidad $x^{*}$.

\subsection{Definición del valor de los pagos de cada coalición}

Para representar el valor de un juego, se utilizará una función característica $v$ con propiedades similares a las que se definen por Von Neumann y Morgenstern para juegos de 
$n$-actores presentadas en las ecuaciones 1,2 y 3 . Donde $\mathbb{B}^{\mathrm{n}}$ es el set de todos los subsets que se pueden formar de los miembros de $\mathbf{N}$.

$$
v: \mathbb{B}^{\mathrm{n}} \rightarrow \mathbb{R}
$$

Esto quiere decir que para cada $\mathbf{T} \subset \mathbf{N}$, el valor de la coalición estará dado por $v(\mathbf{T})$ para una $\mathbf{N}$ finita. Una propiedad interesante de este nuevo método es que permite relajar la superaditividad (ecuación 3), de modo que el valor generado del juego no depende de la creación de valor en cada subcoalición, sino únicamente del valor de la gran coalición (N) con respecto al resto de las subcoaliciones.

$$
v(\mathbf{N}) \geq v(\mathbf{S})+v(\mathbf{T}) \quad \text { si } \quad \mathbf{S} \cap \mathbf{T}=\emptyset, \quad \mathbf{S} \cup \mathbf{T}=\mathbf{N} \quad \text { para } \quad \mathbf{S}, \mathbf{T} \subset \mathbf{N} .
$$

Al ser quasi-superaditiva (ecuación 5) v permite modelar adecuadamente el comportamiento de los pagos del juego al irse incrementando el número de jugadores. De tal forma que $v(\mathbf{N})$ provee mayores beneficios que cualquier otra coalición $\mathbf{S} \subset \mathbf{N}$ y, en consecuencia, cada jugador obtiene un beneficio mayor o igual al que hubiera obtenido en una coalición compuesta de menos miembros.

\subsection{Definición del Core del juego}

Para definir el Core del juego, basta con utilizar las ecuaciones de Eficiencia (ecuación 6) y Racionalidad (ecuación 7).

$$
\begin{aligned}
& \sum_{i \in \mathbf{N}} x_{i}=v(\mathbf{N}) \\
& \sum_{i \in \mathbf{S}} x_{i} \leq v(\mathbf{S}) \quad \forall \mathbf{S} \subset \mathbf{N} .
\end{aligned}
$$

\subsection{Generación de una muestra representativa $M$ del Core en tér- minos monetarios}

Utilizando JDSIM se puede conocer un número suficientemente grande de negociaciones que cumplan con las restricciones del Core y permitan caracterizarlo relativamente rápido. El número mínimo de muestras requeridas se estima utilizando fair-convergence Montiel and Bickel (2013). Para mayor detalle sobre JDSIM, se invita al lector a revisar Montiel and Bickel (2013).

\subsection{Obtención de las funciones empíricas de probabilidad acumu- lada de cada jugador y la Función de Probabilidad de Ocurrencia}

Una vez obtenida la muestra aleatoria uniformemente distribuida del Core, se modela el comportamiento de los pagos que recibe cada jugador $i$ en la $j$-esima muestra de $\mathbf{M}$ a través de la función empírica de probabilidad acumulada $F_{i} \forall x \in \mathbf{M}$.

$$
F_{i}\left(x_{i}\right)=\frac{1}{m} \sum_{j=1}^{m} \mathbf{1}_{\left\{x_{i}^{j} \leq x_{i}\right\}} \quad \forall i \in \mathbf{N},
$$

donde $\mathbf{1}_{\left\{x_{i}^{j} \leq x_{i}\right\}}$ es una función indicadora definida de la siguiente forma:

$$
\mathbf{1}_{\left\{x_{i}^{j} \leq x_{i}\right\}}=\left\{\begin{array}{ll}
1 & x_{i}^{j} \leq x_{i} \\
0 & \text { en otro caso }
\end{array} .\right.
$$

Esta función permite calcular la probabilidad de que un jugador $i$ reciba un pago menor o igual a un cierto pago $x_{i}$ con base en las estrategias que componen $\mathbf{M}$. 


\subsubsection{La Función de Probabilidad de Ocurrencia}

Una vez obtenidas las funciones empíricas de los pagos de los $n$ jugadores, se propone seleccionar aquella negociación $x^{*}$ que maximice la probabilidad de que la negociación ocurra entre los $n$ jugadores $(P P(x))$. Para el caso de ganancias, se define $P P(x)$ como:

$$
P P(x)=\prod_{i=1}^{n} F_{i}\left(x_{i}\right), \quad \text { donde } \quad x=\left\{x_{1}, x_{2}, \ldots, x_{n}\right\}
$$

y para el caso de costos, se define $\mathrm{PP}(\mathrm{x})$ como:

$$
P P(x)=\prod_{i=1}^{n}\left(1-F_{i}\left(x_{i}\right)\right), \quad \text { donde } \quad x=\left\{x_{1}, x_{2}, \ldots, x_{n}\right\}
$$

Para que se realice una negociación, cada actor necesita aceptar recibir un pago $x_{i}$. Por ello, la probabilidad de aceptar es equivalente a la probabilidad de obtener un beneficio menor si la negociación se repitiera. Es decir, si para una negociación, la probabilidad de que un jugador reciba un pago mayor al que está recibiendo es de $P P=0,4$, entonces, la probabilidad de que acepte ese pago corresponde a la probabilidad de obtener un beneficio menor si volviera a realizarse la negociación que equivale a $P P=0,6$. Por esta razón, si se asume que cada actor decide aceptar o rechazar, considerando únicamente su beneficio económico, la probabilidad de que una negociación se concluya con éxito es igual al producto de las probabilidades de aceptar de cada actor.

\subsection{Determinación del problema de maximización}

Una vez determinada la Función de Probabilidad de Ocurrencia, se puede definir el problema que maximice $P P(x)$, con base en las restricciones que definen el Core del juego.

$$
\begin{array}{lll}
\text { Max } & P P(x), \\
\text { s.a } & \sum_{i \in \mathbf{S}} x_{i} \leq v(\mathbf{S}) & \forall \mathbf{S} \subset \mathbf{N}, \\
& \sum_{i \in \mathbf{N}} x_{i}=v(\mathbf{N}), & \\
& x_{i} \geq 0 & \forall i \in \mathbf{N} .
\end{array}
$$

A partir de la resolución del problema de maximización, se obtiene el vector $x^{*} \in$ $\mathbb{R}$ que especifica la distribución de los pagos que maximiza la probabilidad de que la negociación suceda.

\section{Validación del modelo}

Para validar el modelo, se simularon 30 escenarios aleatorios que replican el ejemplo ilustrativo de la sección §??, en los que la Gran Coalición pudiese ser alcanzada. Se acomodaron de modo aleatorio las direcciones de $n$ actores partiendo de un punto de origen común, asumiendo que cada actor quiere llegar desde un origen $\mathrm{O}$ a su destino. Cada actor pagará un monto equivalente a la distancia recorrida, pero pueden generar un beneficio adicional si viajan juntos. Para cada escenario, se calcularon las distancias entre los destinos finales y se calcularon las rutas óptimas para cada subcoalición. En cada caso, se calculó la solución generada por SV, el Centroide del Core, y la estrategia generada por $P P(x)$.

La Figura 3 muestra la probabilidad de cerrar una negociación para los escenarios considerados. Se puede observar que el nuevo método siempre genera estrategias que proveen un mayor incentivo para que los $n$ actores acepten el pago que se les propone. 
Figura 3. Comparativo de SV, el Centroide del Core y la Negociación de Máxima Probabilidad para diez escenarios generados de modo aleatorio considerando 3 , 4, y 5 actores.
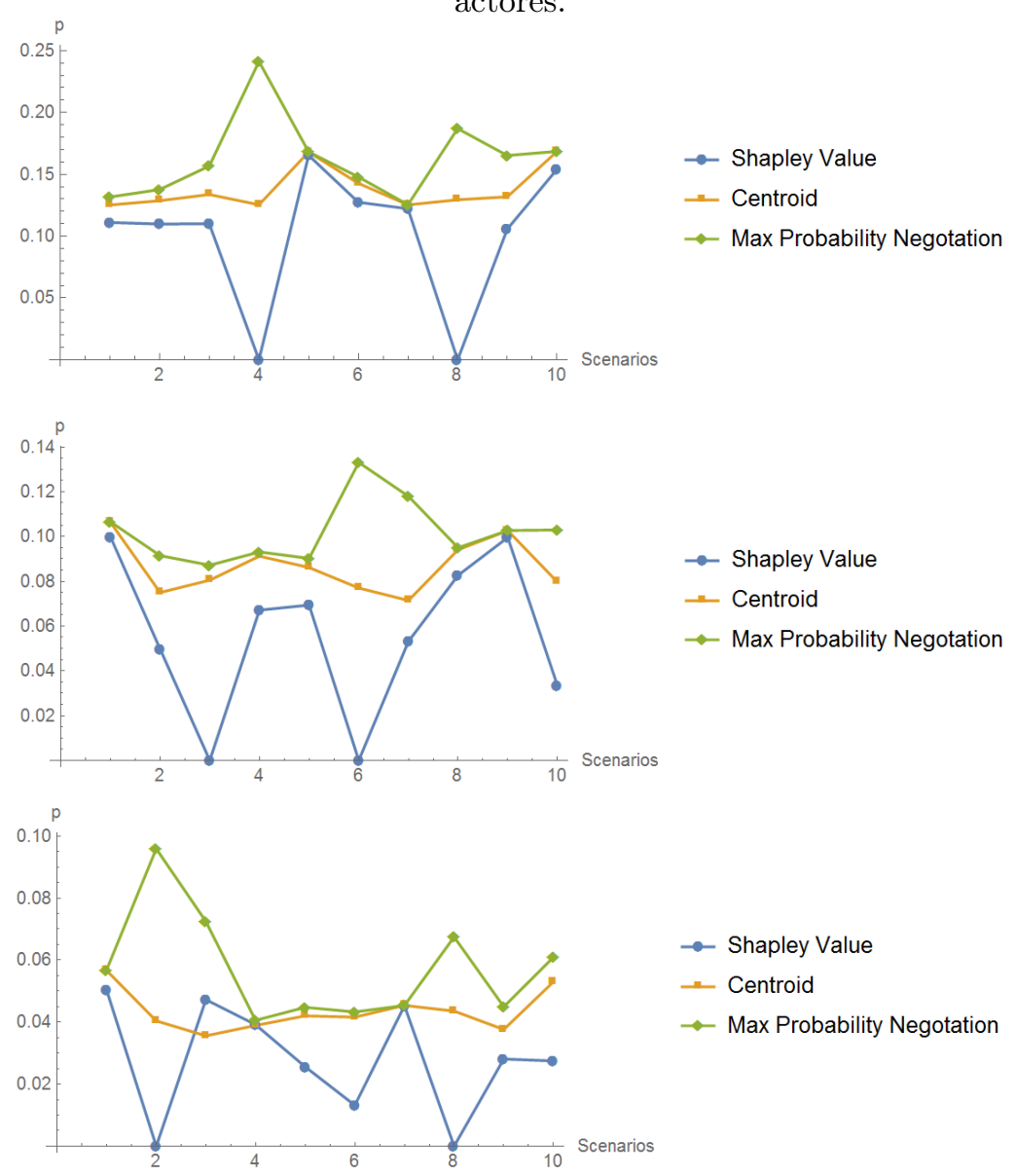

Se puede observar que para seis escenarios, SV genera estrategias para las que $P P(S V)=$ 0 . Esto sucede cuando la estrategia propuesta por SV está fuera del Core, por lo que no es implementable. De modo similar, existen escenarios en los que los tres métodos tienen una $P P(\cdot)$ muy parecida. Esto se explica por la existencia de un Core muy reducido con la estructura de un juego convexo que asegura $\mathrm{SV} \in$ Core. Sin embargo, es claro que nuestro método genera consistentemente estrategias que superan las propuestas por alternativas anteriores.

A manera de recapitulación, se presenta la Tabla 4, que muestra un comparativo de las distintas metodologías y sus características principales. En el comparativo, las palabras implementable y única corresponden a definiciones anteriores, y justificable se refiere a proveer un justificación racional para la selección de la estrategia correspondiente. Adicionalmente, la palabra certificado corresponde a una prueba de la inexistencia de la Gran Coalición, y la palabra solución se refiere al producto final cuando se aplica el método correspondiente. 
Tabla 4. Cuadro comparativo de estrategias de negociación comercial.

\begin{tabular}{|l|c|c|c|c|c|}
\hline & \multicolumn{3}{|c|}{$\begin{array}{c}\text { Si existe la Gran Coalición, } \\
\text { siempre ofrece una solución }\end{array}$} & \multicolumn{2}{c|}{$\begin{array}{c}\text { Si no existe la Gran Coalición, } \\
\text { el método regresa un }\end{array}$} \\
\hline Estrategia de Negociación & Implementable & única & Justificable & Certifcado & Solución \\
\hline Negociación de Máx. Prob. & Sí & Sí & Sí & Sí & No \\
Shapley Value & No & Sí & Sí & No & Incorrecta \\
El Core & Sí & No & No & Sí & No \\
El Centroide del Core & Sí & Sí & No & No & No \\
\hline
\end{tabular}

\section{Caso de estudio}

Finalmente, como una muestra de la aplicabilidad de esta metodología en negociaciones comerciales, se analiza un Caso de Estudio con respecto a la distribución de ganancias en un escenario de racaudación de capital para una startup $(\mathrm{S})^{2}$.

Este escenario de negociación consta de 3 actores $(N=3)$ : la startup $\mathrm{S}$, un inversionista independiente (I) y una segunda startup denominada $(\mathrm{O})$.

$\mathrm{S}$ es una startup mexicana que se dedica a ayudar a migrantes mexicanos en Estados Unidos para que construyan una casa para sus familias en México, vinculándolos con empresas constructoras que tengan la capacidad para hacerlo. S estima que requiere de una inversión en capital de por lo menos $\$ 500,000$ MXN para poder invertir en el desarrollo de su imagen corporativa, su estrategia digital y la construcción de su plataforma tecnológica. Con esta inversión, se estima que el valor presente neto de los flujos libres de efectivo de los próximos cinco años de S sea de $\$ 20,000,000$ MXN en 2018.

I es un inversionista independiente, conocedor del tema de vivienda y dueño de una empresa constructora en México con la capacidad de disponer de $\$ 3,000,000$ MXN para invertir en proyectos de vivienda en México.

O es una startup mexicana que busca homologar las transacciones monetarias en línea que se realicen a través de distintas plataformas digitales. O requiere de una inversión en capital de mínimo $\$ 2,500,000$ MXN para cubrir sus pasivos relacionados con el desarrollo de su software y cubrir el diseño y desarrollo de tarjetas de pre-pago para uso de su plataforma. Con esta inversión, se pronostica que el valor presente neto de los flujos libres de efectivo de O sea de $\$ 100,000,000$ MXN en 2018.

Para esta negociación, I propone invertir $\$ 3,000,000 \mathrm{MXN}$ en $(\mathrm{O})$ para adquirir el $10 \%$ de la participación accionaria de esa empresa y que corresponde a una valuación de $\$ 30,000,000$ MXN de O al día de hoy. De estos tres millones que invierte I, O se compromete a invertir $\$ 500,000$ MXN para adquirir el $10 \%$ de las acciones de $\mathrm{S}$ y además accede a colocar las viviendas que promueve $\mathrm{S}$ en su plataforma digital, por lo que la valuación de S equivale a $\$ 5,000,000 \mathrm{MXN}$ al día de hoy.

Con esta información, se pueden definir las restricciones para los pagos de las coaliciones de un jugador:

$$
S \geq 5,000,000 \quad I \geq 3,000,000 \quad O \geq 30,000,000
$$

Es decir, S solamente aceptará aquellas negociaciones en las que el valor de la empresa crezca por lo menos, por encima de su valor actual de $\$ 5$ millones; I las que generen ganancias iguales o mayores a $\$ 3$ millones, y $\mathrm{O}$ las que generen un valor mayor o igual a $\$ 30$ millones.

Adicionalmente, se sabe que S está dispuesta a negociar con I para levantar el capital que requiere obteniendo una restricción de los pagos que debe ser mayor o igual a $\$ 19,000,000$, que es el valor estimado que valdría la empresa por la inversión que reciba de I, pero sin las posibles sinergias que podría haber formado con $\mathrm{O}$.

$$
S+I \geq 19,000,000
$$

\footnotetext{
${ }^{2}$ Hérita S.A de C.V.
} 
Sin embargo, S no está dispuesta a participar en una negociación comercial en la que la única contraparte sea $\mathrm{O}$. Esto es debido a que a $\mathrm{S}$ no le interesa adquirir una participación accionaria en $\mathrm{O}$ ni tampoco que $\mathrm{O}$ se vuelva accionista de $\mathrm{S}$, pues $\mathrm{O}$ no le puede ofrecer a $\mathrm{S}$ el expertise que un inversionista como I le puede ofrecer. Por esta razón la coalición entre S y O no puede ocurrir, y se fija en un valor de $\$ 35,000,000$ MXN cantidad que cada jugador ganaría como mínimo si cada uno actuara por su cuenta.

$$
S+O \geq 35,000,000
$$

Ahora bien, en teoría, la coalición entre I y O no podría ocurrir al igual que la coalición anterior. Esto obedece a que $\mathrm{S}$ fue el que invitó a I a unirse a la negociación con O, por lo que implícitamente I aceptó participar en la negociación siempre y cuando S estuviera en la negociación. No obstante, S sabe que I y O podrían eliminarla de la negociación y formar una coalición por su cuenta en la que el valor de O aumentaría a \$98,000,000 MXN.

$$
I+O \geq 98,000,000
$$

Sin embargo, si I decide invertir en ambas empresas, se podrían generar sinergias entre estos 3 actores y el valor de cada empresa aumentaría, de tal forma que el valor total que se podrían repartir sería de $\$ 120,000,000$ MXN.

$$
S+I+O \geq 120,000,000
$$

Con base en esta información, se resumen todas las coaliciones que componen el Core del juego en la Tabla 5:

Tabla 5. Escenario de Negociación

\begin{tabular}{lr}
\hline \hline Coalición & Valor del Juego \\
\hline$(\mathrm{S})$ & $\$ 5,000,000$ \\
$(\mathrm{I})$ & $\$ 3,000,000$ \\
$(\mathrm{O})$ & $\$ 30,000,000$ \\
$(\mathrm{~S}, \mathrm{I})$ & $\$ 19,000,000$ \\
$(\mathrm{~S}, \mathrm{O})$ & $\$ 35,000,000$ \\
$(\mathrm{I}, \mathrm{O})$ & $\$ 98,000,000$ \\
$(\mathrm{~S}, \mathrm{I}, \mathrm{O})$ & $\$ 120,000,000$ \\
\hline
\end{tabular}

Con lo anterior, aplicando esta metodología y calculando las soluciones que ofrecen Shapley Value y el Centroide, se obtienen los siguientes resultados:

Tabla 6. Negociaciones propuestas

\begin{tabular}{|c|c|c|c|}
\hline Jugador & Max Proba & SHV & Centroide \\
\hline S & $22,000,000$ & $12,500,000$ & $13,400,000$ \\
I & $37,230,000$ & $43,000,000$ & $41,610,000$ \\
O & $60,770,000$ & $64,500,000$ & $64,990,000$ \\
\hline \hline PP & 0.193144 & 0.114995 & 0.125741 \\
\hline
\end{tabular}

Como puede observarse en la Tabla 6, la negociación de máxima probabilidad le asigna un pago mayor al jugador S que los pagos que le asignan los métodos SV y el Centroide, aumentando la probabilidad de que la negociación ocurra.

Al inicio de la negociación, el valor agregado de los jugadores $\mathrm{S}$, I y O es de $\$ 38$ millones ( $\$ 5$ millones de S, $\$ 30$ millones de O y $\$ 3$ millones de I), pero al cooperar y formar la Gran Coalición, el valor total generado es de $\$ 120$ millones, produciendo así $\$ 82$ millones adicionales. De acuerdo con la negociación de máxima probabilidad, este 
valor adicional generado debe repartirse de la siguiente forma: $\$ 22$ millones para S, que implica una ganancia de $\$ 17$ millones con respecto a su valor inicial; $\$ 37.23$ millones para I, que equivale a $\$ 34.23$ millones de ganancia, y $\$ 60.77$ millones para $\mathrm{O}$, que corresponde a un incremento de $\$ 30.77$ millones con respecto a su valor inicial. Como se aprecia en la Tabla 7, cada uno de los 3 jugadores gana más de lo que ganaría si la negociación no se concreta; de hecho, la ganancia para cada uno es:

Tabla 7. Beneficio para cada jugador por participar en la Gran Coalición

\begin{tabular}{|c|c|c|c|}
\hline & S & I & O \\
\hline Ganancia (MXN) & $17,000,000$ & $34,230,000$ & $30,770,000$ \\
Crecimiento (\%) & $340 \%$ & $1,141 \%$ & $102.56 \%$ \\
\hline
\end{tabular}

Ahora bien, por la simple razón de que cada uno de los jugadores gana una cantidad mayor a la que tiene actualmente, debería ser del interés de todos que la negociación se concrete. Nótese que las valuaciones iniciales de S y O de $\$ 20$ millones y $\$ 100$ millones solamente variaron en $\$ 2$ millones que fueron transferidos al valor de S. Esto significa que, si a S le interesa que la negociación ocurra, debe ofrecer $\$ 37.23$ millones a I, $\$ 60.77$ millones a $\mathrm{O}$ y quedarse únicamente con $\$ 22$ millones de los $\$ 120$ millones que se pueden generar al cooperar entre sí. Es decir, si S busca convencer a I de que invierta en $\mathrm{O}$ e indirectamente en $\mathrm{S}$, además del valor generado por el expertise de I y las sinergias con O, debe aceptar que $\$ 98$ millones de los $\$ 120$ millones disponibles se los dividan estos otros dos actores. Con este escenario, S estaría capturando $\$ 2$ millones adicionales a su estimación inicial, mientras que el valor de $\mathrm{O}$ bajaría por esta misma cantidad.

En consecuencia, la recomendación que proporciona esta metodología para S en su negociación con I y O es que busque capturar $\$ 22$ millones, en lugar de $\$ 20$ millones de los $\$ 120$ millones disponibles, ofreciendo a I y O un pago de $\$ 37.23$ millones y $\$ 60.77$ millones respectivamente.

\section{Conclusiones}

Este trabajo presenta un método fácil de calcular y con una fuerte justificación racional para desarrollar estrategias de distribución de beneficios en negociaciones comerciales de $n$ actores. Dados los experimentos realizados, este nuevo método supera a los métodos anteriores porque propone una solución única (a diferencia del Core), que es siempre implementable (a diferencia de SV), y que ofrece una fuerte justificación racional (a diferencia del Centroide del Core). Esto se logra gracias a la implementación de JDSIM que permite generar un réplica discreta del Core para crear las funciones empíricas de probabilidad acumulada de cada actor, de modo que cualquier solución fuera del Core tiene probabilidad cero de ser considerada. Por ello, esta propuesta siempre ofrece una solución dentro del Core (implementable) y proporciona una fuerte justificación al integrar las distribuciones empíricas de cada actor para encontrar la estrategia que maximiza la probabilidad conjunta de los $n$ actores de formar la Gran Coalición.

La flexibilidad de este método le permite adaptarse a negociaciones reales como se vio en el Caso de Estudio y ofrecer soluciones que sean ejecutables y tengan un racional fuerte. Adicionalmente, el método puede incluir o remover restricciones con facilidad e incluir funciones de utilidad que ayudan a compensar la aversión al riesgo de los distintos actores.

Se debe mencionar que, una limitante de este trabajo es que propone estrategias de negociación solamente en escenarios en los que se puede formar la Gran Coalición. Sin embargo, plantea un nuevo paradigma para extender la literatura en Teoría de Juegos Cooperativos por medio de nuevos algoritmos que usen este método como base para crear estrategias de negociación en subcoaliciones que tengan un Core no vacío, y de esta manera puede generar posibles alternativas al Bargaining Set y otros métodos relacionados. 
Actualmente, se está trabajando en aspectos de análisis en relación con este nuevo método, y en mejorar la eficiencia del algoritmo para hacerlo práctico y fácil de utilizar en instancias de mayor tamaño. No obstante, se tiene confianza que, en la práctica, los negociadores encontrarán esta nueva herramienta extremadamente útil, y los académicos encontrarán un nuevo campo de estudio para hacer desarrollos en Teoría de Negociaciones.

\section{Agradecimientos}

Este trabajo fue apoyado por el Consejo Nacional de Ciencia y Tecnología de México (CONACYT), y el Sistema Nacional de Investigadores (SNI) bajo la concesión de Luis V. Montiel número 259968.

Adicionalmente, se agradece el apoyo de la empresa Hérita S.A de C.V por proporcionarnos los datos e información necesaria para analizar el escenario de negociación en la sección del Caso de Estudio.

\section{Referencias}

Aumann, R. J. and Maschler, M. (1961). The bargaining set for cooperative games, volume 10 of Econometric Research Program. Princeton University, Princeton, N.J.

Davis, M. and Maschler, M. (1965). The kernel of a cooperative game. Naval Research Logistics (NRL), $12(3): 223-259.14$

Devroye, L. (1986). Non-uniform random variate generation, volume 1 of Probability Theory and Stochastic Processes. Springer-Verlag New York, New York, september edition.

Edgeworth, F. Y. (1881). Mathematical psychics: An essay on the application of mathematics to the moralsciences, volume 10. C. Kegan Paul and co.

Gillies, D. B. (1959). Solutions to general non-zero-sum games. Contributions to the Theory of Games, $4(40): 47-85$.

Lucas, W. F. (1969). The proof that a game may not have a solution. Transactions of the American Mathematical Society, 137(1):219-229.

Lucas, W. F. (1992). Chapter 17 von neumann-morgenstern stable sets. In Handbook of Game Theory with Economic Applications, volume 1, chapter Chapter 17: von Neumann-Morgenstern stable sets, pages543-590. Elsevier.

Montiel, L. V. and Bickel, J. E. (2013). Generating a random collection of discrete joint probability distri-butions subject to partial information. Methodology and Computing in Applied Probability, 15(4):951-967.

Rubin, P. A. (1984). Generating random points in a polytope. Communications in Statistics - Simulationand Computation, 13(3):375-396.

Schmeidler, D. (1969). The nucleolus of a characteristic function game. SIAM Journal on applied mathematics, 17(6):1163-1170.

Shapley, L. S. (1952). Notes on the n-person game, III: Some variants of the von Neumann-Morgenstern definition of solution, volume 10. Rand Corporation, Santa Monica, CA.

Shapley, L. S. (1953). A value for n person games. Contributions to the Theory of Games, 2(28):307-317.

von Neumann, J. (1963). Various techniques used in connection with random digits, volume 5 ofvon Neu-mann's Collected Works. Pergamon Press, Oxford.

von Neumann, J. and Morgenstern, O. (1944). Theory of Games and Economic Behavior, volume 1. Princeton University Press. 